\title{
Research The Representativeness of An Isolated Medial Malleolar Fracture
}

Chi-Chuan Wu ( $\nabla$ ccwu@mail.cgu.edu.tw)

Chang Gung Memorial Hospital https://orcid.org/0000-0003-3609-7986

\author{
Wen-Ling Yeh \\ Chang Gung Memorial Hospital \\ Po-Cheng Lee \\ Chang Gung Memorial Hospital

\section{Ying-Chao Chou} \\ Chang Gung Memorial Hospital

\section{Yung-Heng Hsu} \\ Chang Gung Memorial Hospital

\section{Yi-Hsun Yu} \\ Chang Gung Memorial Hospital
}

\section{Research}

Keywords: Fracture, Isolated, Medial malleolus, Representativeness

Posted Date: May 14th, 2020

DOl: https://doi.org/10.21203/rs.3.rs-27976/v1

License: (c) (i) This work is licensed under a Creative Commons Attribution 4.0 International License. Read Full License 


\section{Abstract}

Background: An isolated medial malleolar fracture (IMMF) is relatively uncommon.

Not only is the treatment principle undetermined well, but also is the patho-mechanism debatable. The goal of this retrospective study intended to clarify the patho-mechanism of an IMMF and develop an optimal treatment principle to upgrade a success rate.

Methods: For the 10-year period, 59 consecutive adult patients (average, 38 years; range, 18-77 years) with an IMMF were treated. The causes included $42.4 \%$ of motorcycle accidents, $20.3 \%$ of working injuries, $10.2 \%$ of car accidents, $10.2 \%$ of slide injuries, and $16.9 \%$ of others. The 59 injuries were classified to $59.3 \%$ of stage 1 pronation-external rotation (PE), $20.3 \%$ of stage $2 \mathrm{PE}, 10.2 \%$ of stage 2 pronation-abduction (PA), and $10.2 \%$ of stage 2 supination-adduction (SA) injuries. Unstable fractures which comprised stage 2 PA and stage 2 SA injuries were treated with internal fixation. Stable fractures which comprised stage $1 \mathrm{PE}$ and stage $2 \mathrm{PE}$ injuries were treated with either internal fixation or conservative techniques.

Results: Fifty patients (84.7\%, 50 / 59) were followed for at least one year (average, 1.3 years; range, 1-3.1 years). Forty-nine fractures healed with a union rate of $98 \%$. Satisfactory ankle function was achieved in $84 \%$ of patients (42 / 50). Four out of eight patients with an unsatisfactory ankle function sustained syndesmotic diastasis. One fracture which was treated with internal fixation sustained screw breakage with nonunion. This patient also had unsatisfactory ankle function.

Conclusion: Not all IMMFs can be treated conservatively. Practically, an IMMF should be classified into a stable or unstable type based on radiographs and clinical stress tests. A stable type may include $80 \%$ of IMMFs which may be treated with either surgical or conservative techniques. An unstable type may include $20 \%$ of IMMFs which had better be treated with internal fixation.

\section{Background}

Ankle injuries are common in the daily activity. Clinically, most of ankle injuries are not severe and usually can be treated with various non-surgical methods with a success [1, 2]. Because of the unfavorable bony structures (the huge distal tibia with the small lateral malleolus), the stresses transfer inside the ankle is relatively unsmooth $[3,4]$. Additionally, the foot is limited to a unidirectional movement (dorsi- and plantar flexion) [5]. Once various directions of ground reaction forces are applied on the ankle, varied complex injuries will happen. Until now, no single classification can be convincingly considered able to include all ankle injuries. Clinically, combined Lauge-Hansen and Weber classifications have been used by many orthopedic surgeons for a guide of treatment $[6,7]$. They believe that the combined classifications may comprise the majority of severe ankle injuries.

The Weber classification supports the treatment based on the level of fibular fractures. The more proximal is a fibular fracture, the severer is damage to syndesmotic ligaments and interosseous 
membrane. The Lauge-Hansen classification focuses on patho-mechanism of foot position and applied stresses during injuries. Fibular fractures will happen in advanced stages of ankle injuries [6, 7]. In the literature, various studies related to ankle injuries with the lateral malleolus or fibular fracture are numerous. Nevertheless, despite that some consensuses are achieved, the debates are still ubiquitous.

Studies for medial malleolus fractures are also numerous in the literature. However, the majority of studies focus on comparison of various treatment techniques [8]. Studies for investigating the pathomechanism of an IMMF are relatively few. Based on the Lauge-Hansen classification, an IMMF usually occurs in the initial stage of pronation ankle injury or the advanced stage of supination-adduction (SA) ankle injuries $[6,7,9]$. The pronation injury includes pronation-external rotation (PE) and pronationabduction (PA) types. With more stresses on either pronation injury, syndesmosis injuries will appear. If stresses furthermore act, a fibular fracture will happen. Therefore, an IMMF may or may not associate with syndesmotic instability before a fibular fracture occurs. Treatment of an IMMF without consideration of possible instability of the syndesmosis will run a risk of treatment failure. Clinically, a stage $2 \mathrm{PE}$ ankle injury is considered stable in the syndesmosis and a stage 2 PA injury is unstable in the syndesmosis [6, $7,10]$. Because accurate diagnosis of syndesmotic instability with clinical examinations in the initial stage is not always achievable, understanding of the patho-mechanism of an IMMF may be an important knack. With deep suspicion, the rate of accurate diagnosis may be greatly upgraded. The advanced SA ankle injury is generally not correlated with syndesmotic instability [6, 7]. The primary goal of this retrospective study was to explore how to differentiate an IMMF initiating from what type of ankle injuries. The second goal was to develop the optimal principle for effective treatment of an IMMF.

\section{Methods}

This study had been approved by Institutional Review Board of the authors' institution (IRB: 201900950B0).

\section{Subjects}

From January 1, 2008 to December 31, 2017, 756 consecutive adult patients (> 18 years) with ankle injuries were treated with casting, brace or internal fixation at our institution. Fifty-nine patients had an IMMF were recruited in the present study. Patients aged from 18-77 years (average, 38 years) with a male to female ratio of 3 to 2 . The causes of 59 fractures included motorcycle accident (25), working injuries (12), car accident (6), slide (4), and others (12). The inclusion criteria of this study were adult patients with an IMMF, without prior surgical or non-surgical treatment, and having complete medical records. The exclusion criteria were association with ipsilateral foot crushed injuries or prior deformities.

At the outpatient department (OPD) or emergency service, patients' general conditions were stabilized first. The ankle injuries were examined carefully and classified based on combined Weber and LaugeHansen classifications (Fig. 1) [6, 7]. Non-surgical or surgical treatment of an IMMF was decided immediately (Fig. 2). For a fracture without surgical treatment, a short leg cast or brace was applied for 6 
weeks. It was based on the degrees of ankle swelling. For a fracture requiring surgical treatment, the surgery was arranged as soon as possible. It was performed at an average of 2.4 days later (range, 0.511 days). The surgical necessity was completely decided by an orthopedic surgeon on duty. In principle, an unstable ankle required internal fixation. A stable ankle was operated on based on the degrees of displacement (>2 mm) [11, 12].

Patho-mechanically, a stage 1 PE or PA injury was initially from a transverse or oblique medial malleolar fracture (Fig. 1) [6, 7]. Differentiating the two was basically difficult. Because clinically the incidence of a PE injury was reported three times more common than a PA injury, for statistical convenience all stage 1 PE injuries were used to represent the total of the two types $[10,13]$. Similarly, a stage 2 PA injury was a medial malleolar fracture adding a sole tear of anterior inferior tibiofibular (AITF) ligament, a sole tear of posterior inferior tibiofibular (PITF) ligament or combined tears of both ligaments [14]. If the clue of a tear of PITF ligament could not be pursued (e.g. avulsed fragments on radiographs), a stage 2 PA injury could not be distinguished from a stage $2 \mathrm{PE}$ injury with radiographs. Under such a situation, stress tests (Cotton and external rotation tests) decided a stage 2 PE or PA injury based on stability [15]. To differentiate stage 1 PE from stage 2 PE injuries, avulsion of AITF ligament on radiographs was the key element $[6,7]$. Or, both were represented by stage 1 PE injuries. To differentiate stage 1 PA from stage 2 PA injuries, both radiographs and stress tests were used concomitantly [15]. The former was stable and the latter, unstable $[6,7,10,16]$. At last, in the present study 59 fractures included 35 stage $1 \mathrm{PE}, 12$ stage $2 \mathrm{PE}, 6$ stage $2 \mathrm{PA}$, and 6 stage $2 \mathrm{SA}$ injuries. There were no stage $1 \mathrm{PA}$ injuries which were merged into stage 1 PE injuries. Stage 2 PA and stage 2 SA injuries were unstable. Stage 1 PE and stage 2 PE injuries were stable $[6,7,10,16]$. An unstable injury required internal fixation and a stable injury could be treated with either internal fixation or conservative techniques [16].

\section{Surgical procedure}

Under general anesthesia with endotracheal intubation or spinal anesthesia, the patient was placed on the operating table in the supine position. A pneumatic tourniquet was used routinely and an image intensifier was prepared.

After the skin above the medial malleolus was incised, the fracture fragments were identified. The interposed soft tissues were removed and the fracture fragments were directly reduced. The reduced fragments were stabilized with cancellous screws (Synthes, Bettlach, Switzerland) or Kirschner wires (Mizuho, Tokyo, Japan) under image intensifier guidance. The syndesmosis was not stabilized with a cortical screw despite that an unstable ankle was diagnosed using stress tests (external rotation and hook tests) during surgery [17]. After the wounds were closed, a short splint or brace was used for one month.

Postoperatively, patients were encouraged to ambulate with crutches as early as possible. They were followed at the OPD as the schedule. Clinical and radiographic healing processes were recorded. 
Fracture union was defined as clinically, no pain and tenderness and radiographically, no gaps between the main fracture fragments [18]. Nonunion was defined as the fracture was still unhealed beyond 9 months.

Ankle function was assessed by the American Orthopedic Foot and Ankle Society (AOFAS) score [19]. The total scores were 100 points and included pain, function, and alignment. There were four function grades: excellent (no less than 90 points), good (no less than 80 points), fair (no less than 70 points), and poor (less than 70 points). A satisfactory outcome included an excellent or good grade.

\section{Statistical analysis}

A SPSS version 20 (SPSS Inc., Chicago, IL, USA) software was used for statistical comparison. $\mathrm{P}<0.05$ was considered statistically significant. The chi-square test was used for categorical data and the MannWhitney U test was used for numerical data.

\section{Results}

Fifty patients $(84.7 \%, 50$ / 59) were followed for at least one year (average, 1.3 years; range, 1-3.1 years, Table 1). Nine patients were lost despite all possible efforts to contact them.

Table 1 Comparison of treatment of isolated medial malleolar fractures with varied stability $(\mathrm{n}=50)$

\begin{tabular}{|c|c|c|c|c|c|}
\hline \multirow[t]{2}{*}{ Items } & \multicolumn{2}{|c|}{ Stable } & \multicolumn{2}{|c|}{ Unstable } & \multirow[t]{2}{*}{$p$ value } \\
\hline & PEl & PE2 & PA2 & $\mathrm{SA2}$ & \\
\hline & $(n=30)$ & $(n=11)$ & $(n=6)$ & $(n=3)$ & \\
\hline \multicolumn{6}{|l|}{ Treatment technique } \\
\hline IF: & 23 & 11 & 5 & 2 & \\
\hline Conservative & 7 & 0 & 1 & 1 & \\
\hline Union rate & $97.6 \%$ & $(40 / 41)$ & $100 \%$ & $(9 / 9)$ & 0.23 \\
\hline Syndesmotic diastasis & $4.9 \%$ & $(2 / 41)$ & $11.1 \%$ & $(1 / 9)$ & 0.48 \\
\hline AOFAS score(points) & 81.0 & & 81.7 & & 0.30 \\
\hline Satis factory rate & $87.8 \%$ & $(36 / 41)$ & $66.7 \%$ & $(6 / 9)$ & 0.12 \\
\hline \multicolumn{6}{|c|}{$\begin{array}{l}\text { AOFAS: American OrthopedicFo ot and Ankle Society, I.F.: intemal fixation; } \\
1 \text { pronation-externalrotation; PA2: } 5 \text { tage } 2 \text { pronation-abduction; PE2: stage } 2 \\
\text { pronation-external rotation; SA2: stage } 2 \text { supination-adduction. }\end{array}$} \\
\hline
\end{tabular}

Forty-nine fractures healed within 3 months (a union rate of $98 \%$; Figs. 3-5). One fracture was persistently unhealed with screw breakage at 7 months. Although the ankle function was graded unsatisfactory (score of 78 points), the patient still refused further treatment at 2-year follow-up (Fig. 6).

Satisfactory ankle function was achieved in $84 \%$ of patients (42 / 50). Eight patients with unsatisfactory ankle function included four stage 2 PE injuries (36.4\%, 4 / 11), two stage 2 SA injuries $(66.7 \%, 2$ / 3), one stage 2 PA injury $(16.7 \%, 1 / 6)$, and one stage 1 PE injury $(3.3 \%, 1 / 30)$. 
Three patients had syndesmotic diastasis and all had unsatisfactory ankle function. The three patients included two stage $2 \mathrm{PE}$ and one stage $2 \mathrm{SA}$ injury. Before treatment, all ankles were considered stable [6, $7,10]$. Although all were treated with internal fixation, syndesmotic diastasis still occurred. All patients did not accept further treatment.

Ankle function was compared between stable (stage 1 PE and stage 2 PE injuries) and unstable (stage 2 PA and stage 2 SA injuries) injuries and $p=0.12$ was achieved (satisfactory outcomes, 87.8\% [36/41] versus $66.7 \%[6 / 9]$; post-hoc power $=0.37$ ).

The AOFAS scores were compared between stable and unstable injuries and $p=0.30$ was achieved (81.0 versus 81.7 points).

\section{Discussion}

An IMMF is relatively uncommon in the daily activity. A medial malleolar fracture is easily associated with a lateral malleolar fracture because a supination ankle injury is four times more common than a pronation ankle injury ( $80 \%$ versus $20 \%)[8,12,20,21]$. In normal human gait, the foot sustains the ground reaction forces over the lateral aspect of sole (the medial aspect of sole being vaulted without touching the ground). When a step is missed, the foot is generally in the supine position. The lateral malleolus becomes highly tensed and easily breaks before the medial malleolus $[6,7,20]$.

When a patient with an ankle injury is examined, the whole lateral aspect of the ipsilateral leg should be evaluated concomitantly (Fig. 1) [20, 22]. If pain and tenderness are found, plain radiographs of the tibia must be taken to evaluate a combined fibular fracture. The lateral radiograph of ankle must be carefully inspected to search for a concomitant fracture of the posterior malleolus. If it exists, a stage 2 PA injury may be diagnosed when a medial malleolar fracture also occurs [14]. If it is absent, a stage 1 or $2 \mathrm{PE}$ or PA injury may be possible. Because a stage 2 PA injury is unstable, using internal fixation to stabilize the medial malleolar fracture is imperative [16]. Three other injuries may be treated with non-surgical techniques, e.g. casting or bracing. Practically, differentiating a stage 2 PA injury from three other injuries should utilize clinical stress tests (Cotton and external rotation tests) [15]. Although a stage 2 SA injury without a lateral malleolar fracture (i.e. deltoid ligament tear) is also demonstrated as an IMMF on radiographs, the vertical fracture line is typical for diagnosis $[7,9,23,24]$. Internal fixation is also imperative because it is unstable.

In the literature, a stage 2 PA injury has been reported to possibly exist at one of following situations: a sole tear of the AITF ligament [7, 10], a sole posterior malleolar fracture [6], or combined PITF ligament and these injuries [25]. Therefore, definite diagnosis of a stage 2 PA injury may be difficult and debatable. Theoretically, clinical stress tests should be used to assist decision-making whether conservative or internal fixation treatment should be chosen [11]. Practically, internal fixation should be performed immediately once the suspicion appears. The surgical risk is minimal but the cost-effectiveness is great. This is especially suitable for a stage 2 PA or a stage 2 SA ankle injury combined with an IMMF [16]. 
Currently, stabilization of a displaced medial malleolar fragment has achieved a great success $[8,16,26]$. Various stabilizing techniques generally can be used without problems. Because the medial malleolus is rich of cancellous bone, fracture healing is fast and effective. The possible problem is whether the syndesmosis should be stabilized with a cortical screw in a stage 2 PA injury $[11,26]$. Despite that this doubt is still existed, external rotation stress and hook tests should be utilized after the medial malleolar fragment is stabilized [17]. Insertion of a trans-syndesmotic screw under imaging intensifier guidance may achieve the optimal treatment. In the present study, one of six stage 2 PA injuries had an unsatisfactory outcome. After internal fixation of the medial malleolar fracture, the syndesmosis diastasis occurred. Five other stage 2 PA injuries had no syndesmotic diastasis and had a satisfactory ankle function.

The outcomes of surgical or non-surgical treatment of an IMMF have been reported numerously. Generally, a satisfactory outcome is predictable [12, 27, 28]. In 2018, Hu et al. reported surgical treatment of medial malleolar fractures involving one, two or three malleoli. The nonunion rate was $3.7 \%$ [29]. In 2019, Lokerman et al. underwent a systematic review about a union rate in treatment of an IMMF [22]. The average nonunion rate after surgical treatment was $1.7 \%$ and after conservative treatment, $3.5 \%$. The present study chose either treatment based on syndesmotic stability and achieved a $2 \%$ nonunion rate. To further upgrade the success rate, theoretically the stability should be authentically distinguished. Or, after conservative treatment is finished and the ankle is still unstable, progressive degeneration will be unavoidable $[2,30,31]$.

An IMMF appears in the initial stage of pronation injuries and consequently either stable or unstable ankle may gradually be exposed. Until now, accurate prediction of the most possible pathway based on the first stage is nearly impossible. Practically, by way of various stress tests to assess stability on the spot may be the optimal approach in decision of treatment of an IMMF $[12,16]$.

Limitations of the present study may exist. First, an unstable IMMF cannot be studied with a randomized controlled trial. Therefore, whether an unstable IMMF can be treated conservatively cannot achieve a definite answer. After all, an unstable IMMF is relatively uncommon. In the present study, 12 unstable IMMFs occurred through the 10-year period. Therefore, internal fixation for an unstable IMMF may be the optimal choice. Second, differentiating stage 1 or stage 2 PA injury and differentiating stage 2 PA or stage $2 \mathrm{PE}$ injury deeply base on stress tests. In the literature, various stress tests are considered to have large varieties in sensitivity and specificity [15]. A stable or unstable IMMF may not be reliable. At this moment, any doubt should force the orthopedic surgeon to perform internal fixation [16]. Third, the definite pathological structures in a stage 2 PA injury still cannot be clarified. A simple AITF ligament tear, a simple PITF ligament tear or tears of both ligaments cannot be confirmed [25]. It therefore causes the confusion of a stage 2 PE or a stage 2 PA. The influence is great because of different stability and optimal treatment. In the present study, if a stage 2 PA injury cannot be confirmed (with radiographs and stress tests), all are regarded as stage $2 \mathrm{PE}$ injuries. The treatment techniques may consist of surgical or non-surgical approaches. In the present study, a stage 2 PE injury had a $63.6 \%$ satisfactory rate. 
Misguidance of treating a stage 2 PA injury as a stage 2 PE injury with negligence of syndesmotic instability may be a chief culprit.

\section{Conclusion}

Not all IMMFs can be treated conservatively. Practically, an IMMF should be classified into a stable or unstable type based on radiographs and clinical stress tests. A stable type may include $80 \%$ of IMMFs which may be treated with either surgical or conservative techniques. An unstable type may include $20 \%$ of IMMFs which had better be treated with internal fixation.

\section{Abbreviations}

AITF: anterior inferior tibiofibular; AOFAS:American Orthopedic Foot and Ankle Society; IMMF:isolated medial malleolar fracture; PA:pronation-abduction; PE:pronation-external rotation; PITF:posterior inferior tibiofibular; SA:supination-abduction.

\section{Declarations}

\section{Acknowledgements}

Not applicable.

\section{Author's contributions}

CCW was responsible for study design, data analysis, and writing manuscript. Other five authors were responsible for providing and analyzing data.

\section{Funding}

This study did not receive any funding support.

\section{Availability of data and materials}

All data were available with submission.

\section{Ethics approval and consent to participate}

This study had been approved by Institutional Review Board of the authors' institution (IRB: 201900950B0). Consent to participate was unnecessary. 


\section{Consent for publication}

Not applicable.

\section{Competing interests}

The authors do not have any conflict of interests relevant to this study.

\section{Author details}

All authors are working at the Department of Orthopedic Surgery, Chang Gung Memorial Hospital, Chang Gung University, Taoyuan City, Taiwan.

\section{References}

1. Verhage SM, Schipper IB, Hoogendoorn JM. Long-term functional and radiographic outcomes in 243 operated ankle fractures. J Foot Ankle Res. 2015;8:45.

2. McKeown R, Rabiu AR, Ellard DR, Kearney RS. Primary outcome measures used in interventional trials for ankle fractures: A systematic review. BMC Musculoskelet Disord. 2019;20:388.

3. Norkus SA, Floyd RT. The anatomy and mechanisms of syndesmotic ankle sprains. J Athl Train. 2001;36:68-73.

4. Hermans JJ, Beumer A, de Jong TA, Kleinrensink GJ. Anatomy of the distal tibiofibular syndesmosis in adults: A pictorial essay with a multimodality approach. J Anat. 2010;217:633-45.

5. Wu CC, Shih $\mathrm{CH}$, Chen WJ, Tai CL. Tension-band technique for ankle fusion. Orthopedics. 2001;24:37-40.

6. Okanobo H, Khurana B, Sheehan S, Duran-Mendicuti A, Arianjam A, Ledbetter S. Simplified diagnostic algorithm for Lauge-Hansen classification of ankle injuries. Radiographics. 2012;32:E71-84.

7. Tartaglione JP, Rosenbaum AJ, Abousayed M, DiPreta JA. Classifications in brief: Lauge-Hansen classification of ankle fractures. Clin Orthop Relat Res. 2015;473:3323-8.

8. Ricci WM, Tornetta P, Borrelli J Jr. Lag screw fixation of medial malleolar fractures: A biomechanical, radiographic, and clinical comparison of unicortical partially threaded lag screws and bicortical fully threaded lag screws. J Orthop Trauma. 2012;26:602-6.

9. Ebraheim NA, Weston JT, Ludwig T, Moral MZ, Carroll T, Liu J. The association between medial malleolar fracture geometry, injury mechanism, and syndesmotic disruption. Foot Ankle Surg. 2014;20:276-80.

10. Siegel J, Tornetta P III. Extraperiosteal plating of pronation-abduction ankle fractures. J Bone Joint Surg Am. 2007;89:276-81. 
11. van den Bekerom MPJ, Lamme B, Hogervorst M, Bolhuis HW. Which ankle fractures require syndesmotic stabilization? J Foot Ankle Surg. 2007;46:456-63.

12. Carter TH, Duckworth AD, White TO. Medial malleolar fractures: Current treatment concepts. Bone Joint J. 2019;101-B:512-21.

13. Cabuk H, Celebi F, Imren Y, Dedeoglu SS, Kir MC, Uyamk AF, et al. Compatibility of Lauge-Hansen classification between plain radiographs and magnetic resonance imaging in ankle fractures. J Foot Ankle Surg. 2018;57:712-5.

14. Warner SJ, Fabricant PD, Garner MR, Schottel PC, Helfet DL, Lorich DG. The measurement and clinical importance of syndesmotic reduction after operative fixation of rotational ankle fractures. $J$ Bone Joint Surg Am. 2015;97:1935-44.

15. Sman AD, Hiller $C E$, Refshauge KM. Diagnostic accuracy of clinical tests for diagnosis of ankle syndesmosis injury: A systematic review. Br J Sports Med. 2013;47:620-8.

16. van Dijk CN, Longo UG, Loppini M, Florio P, Maltese L, Ciuffreda M, et al. Conservative and surgical management of acute isolated syndesmotic injuries: ESSKA-AFAS consensus and guidelines. Knee Surg Sports Traumatol Arthrosc. 2016;24:1217-27.

17. Pakarinen $\mathrm{H}$, Flinkkila T, Ohtonen P, Hyvonen P, Lakovaara M, Leppilahti J, et al. Intraoperative assessment of the stability of the distal tibiofibular joint in supination-external rotation injuries of the ankle: sensitivity, specificity, and reliability of two clinical tests. J Bone Joint Surg Am. 2011;93:2057-61.

18. Hsu YT, Wu CC, Lee WC, Fan KF, Tseng IC, Lee PC. Surgical treatment of syndesmotic diastasis: emphasis on effect of syndesmotic screw on ankle function. Int Orthop. 2011;35:359-64.

19. Kitaoka HB, Alexander IJ, Adelaar RS, Nunley JA, Myerson MS, Sanders M. Clinical rating systems for the ankle-hindfoot, midfoot, hallux, and lesser toes. Foot Ankle Int. 1994;15:349-53.

20. Hinds RM, Schottel PC, Berkes MB, Little MT, Helfet DL, Lorich DG. Evaluation of Lauge-Hansen designation of Weber $C$ fractures. J Foot Ankle Surg. 2014;53:434-9.

21. Hockenbury TR. Fractures and dislocations of the foot and ankle. In: Placzek JD, Boyce DA, editors. Orthopedic Physical Therapy Secrets. 3rd ed. St. Louis: Elsevier; 2017. pp. 601-8.

22. Lokerman RD, Smeeing DPJ, Hietbrink F, van Heijl M, Houwert RM. Treatment of a scientifically neglected ankle injury: The isolated medial malleolar fracture. A systematic review. J Foot Ankle Surg. 2019;58:959-68.

23. Dumigan RM, Bronson DG, Early JS. Analysis of fixation methods for vertical shear fractures of the medial malleolus. J Orthop Trauma. 2006;20:687-91.

24. Wegner AM, Wolinsky PR, Robbins MA, Garcia TC, Maitra S, Amanatullah DF. Antiglide plating of vertical medial malleolus fractures provides stiffer initial fixation than bicortical or unicortical screw fixation. Clin Biomech. 2016;31:29-32.

25. Warner SJ, Garner MR, Hinds RM, Helfet DL, Lorich DG. Correlation between the Lauge-Hansen classification and ligament injuries in ankle fractures. J Orthop Trauma. 2015;29:574-8. 
26. Carter TH, Mackenzie SP, Bell KR, Hollyer MA, Gill EC, MacDonald DJ, et al. Selective fixation of the medial malleolus in unstable ankle fractures Injury. 2019;50:983-9.

27. Herscovici D, Scaduto JM, Infante A. Conservative treatment of isolated fractures of the medial malleolus. J Bone Joint Surg Br. 2007;89:89-93.

28. Wang X, Zhang C, Yin JW, Wang C, Huang JZ, Ma X, et al. Treatment of medial malleolus or pure deltoid ligament injury in patients with supination-external rotation type IV ankle fractures. Orthop Surg. 2017;9:42-8.

29. Hu J, Zhang C, Zhu K, Zhang L, Wu W, Cai T, et al. Adverse radiographic outcomes following operative treatment of medial malleolar fractures. Foot Ankle Int. 2018;39:1301-11.

30. Segal G, Elbaz A, Parsi A, Heller Z, Palmanovich E, Nyska M, et al. Clinical outcomes following ankle fractures: a cross-sectional observational study. J Foot Ankle Res. 2014;7:50.

31. Michelson JD, Wright M, Blankstein M. Syndesmotic ankle fractures. J Orthop Trauma. 2018;32:104.

\section{Figures}
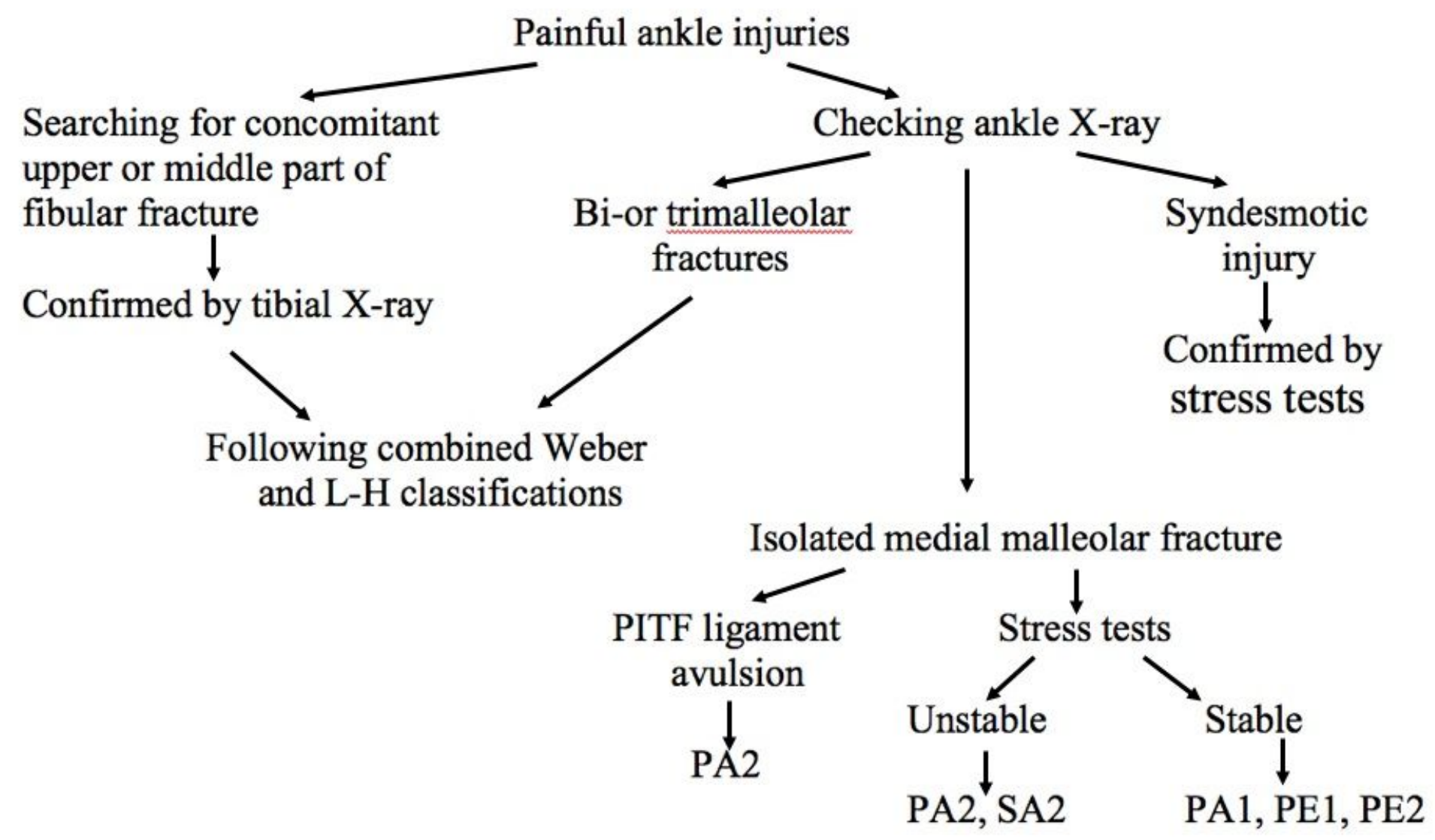

Figure 1 
Algorithm for classification of an isolated medial malleolar fracture. L-H: Lauge-Hansen; PA2: stage 2 pronation-abduction; PE1: stage 1 pronation-external rotation; PE2: stage 2 pronation-external rotation; PITF: posterior inferior tibiofibular; SA2; stage 2 supination-adduction.

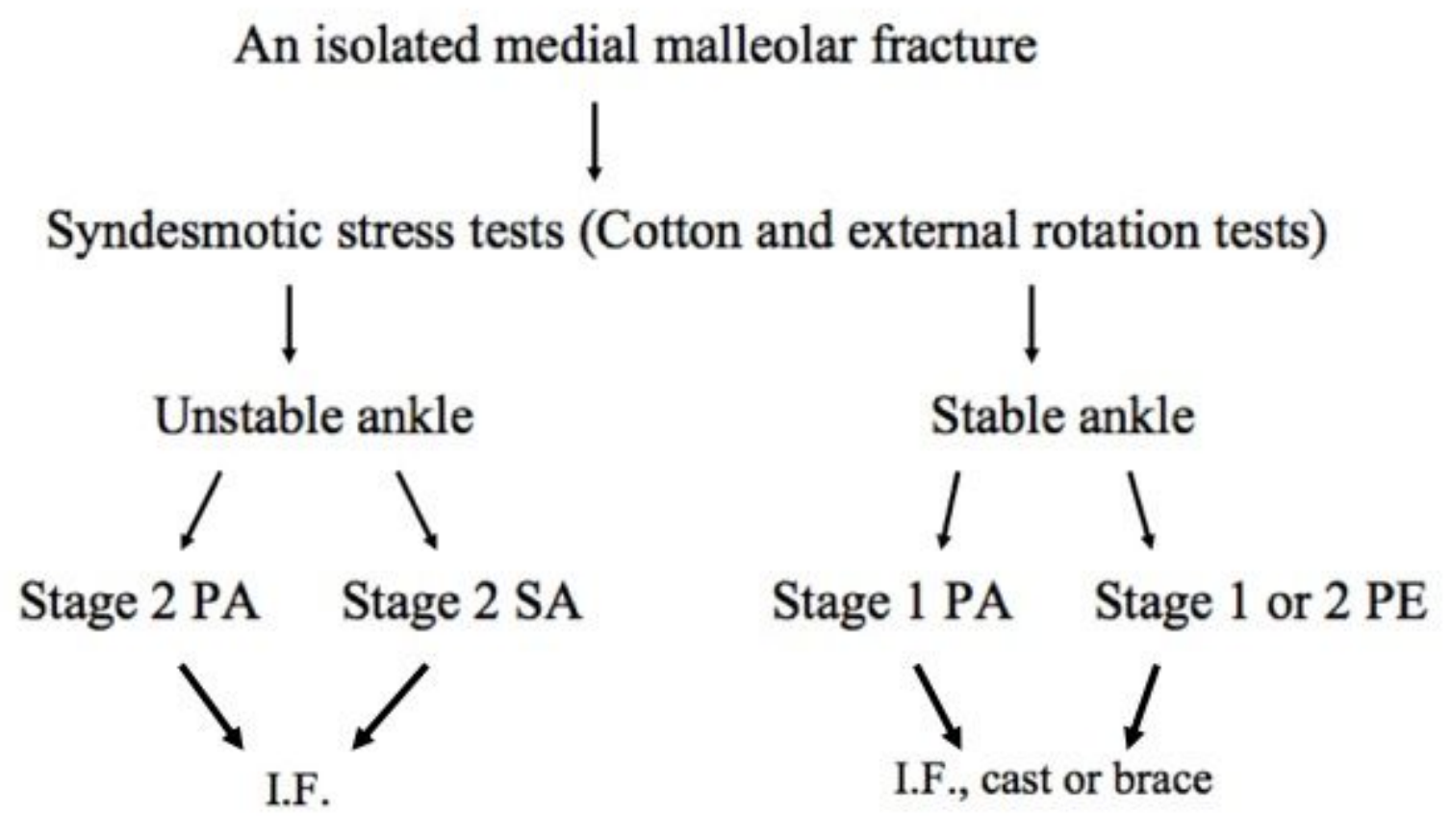

Figure 2

Algorithm for optimal treatment of an isolated medial malleolar fracture. I.F.: internal fixation; PA: pronation-abduction; PE: pronation-external rotation; SA; supination-adduction. 


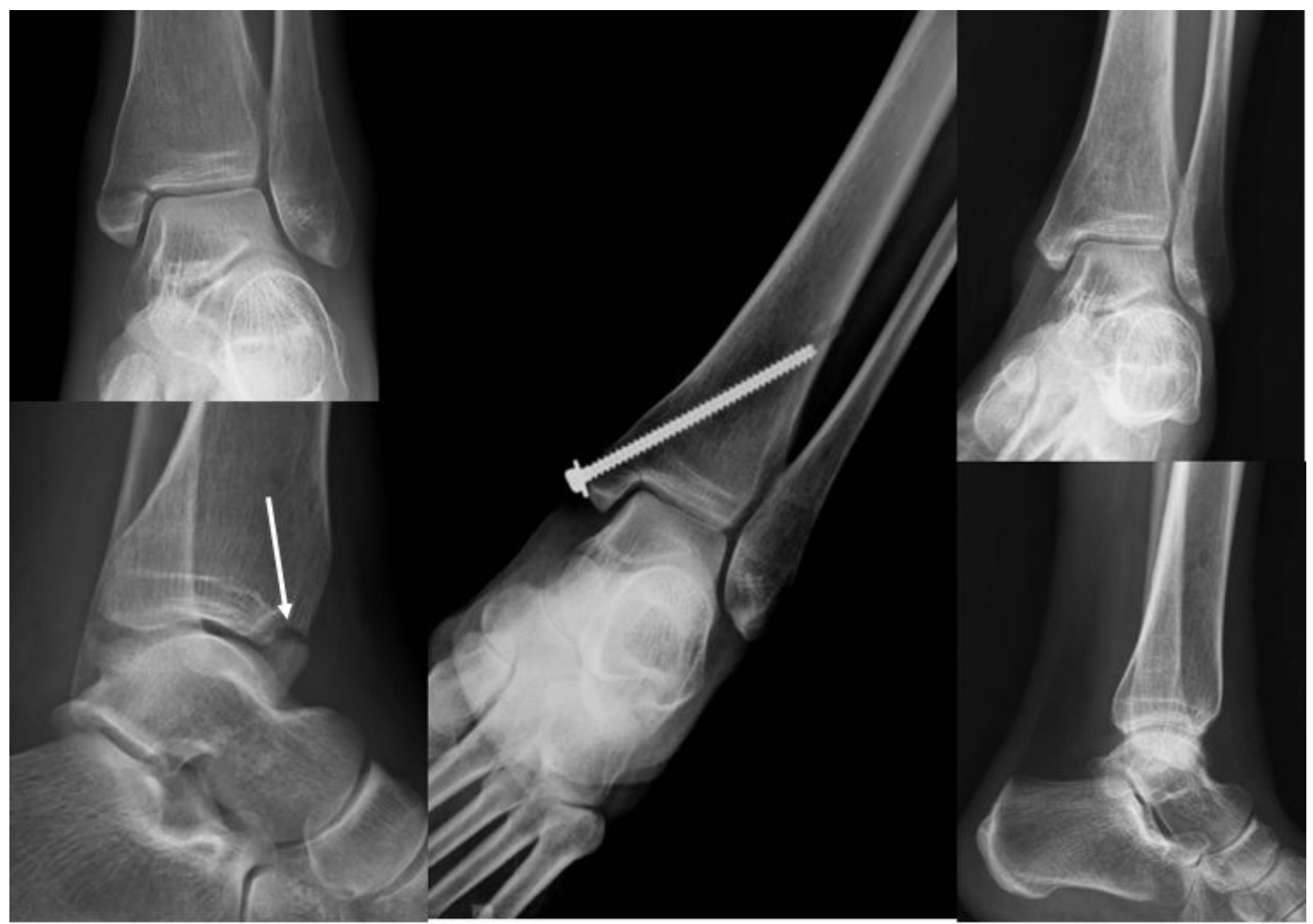

Figure 3

A 54-year-old woman sustained a left isolated medial malleolar fracture due to car accident. A stage 2 pronation-external rotation type was diagnosed based on the avulsion tear of anterior inferior tibiofibular ligament (white arrow). Cancellous screws were inserted for stabilizing fractured medial malleolus. A good ankle function was achieved for 1.5-year follow-up. 


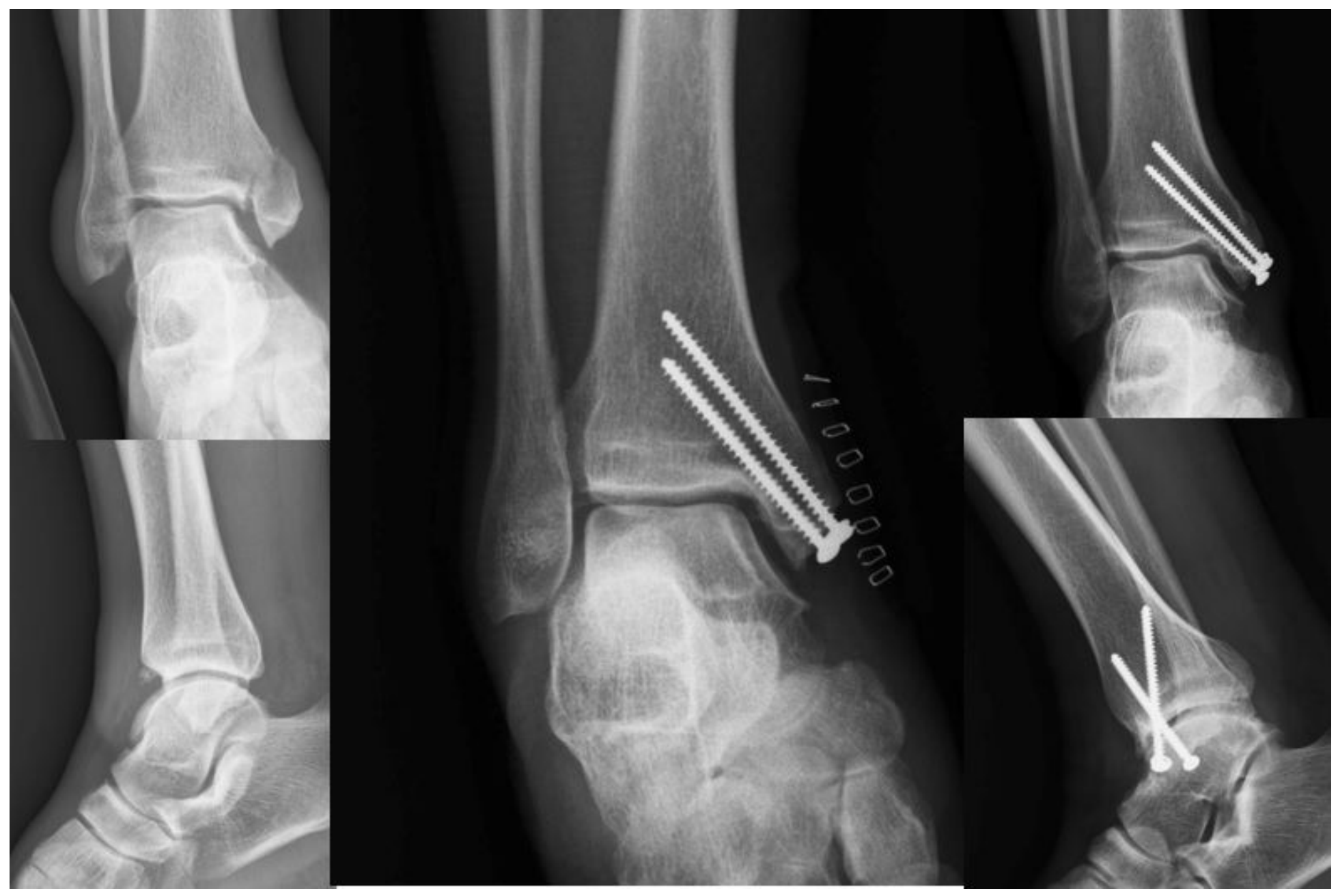

\section{Figure 4}

A 21-year-old woman sustained a right isolated medial malleolar fracture due to bicycle accident. A stage 2 supination-adduction type was diagnosed based on vertical fracture line. Cancellous screws were inserted for stabilizing fractured medial malleolus. A good ankle function was achieved for 1-year followup. 


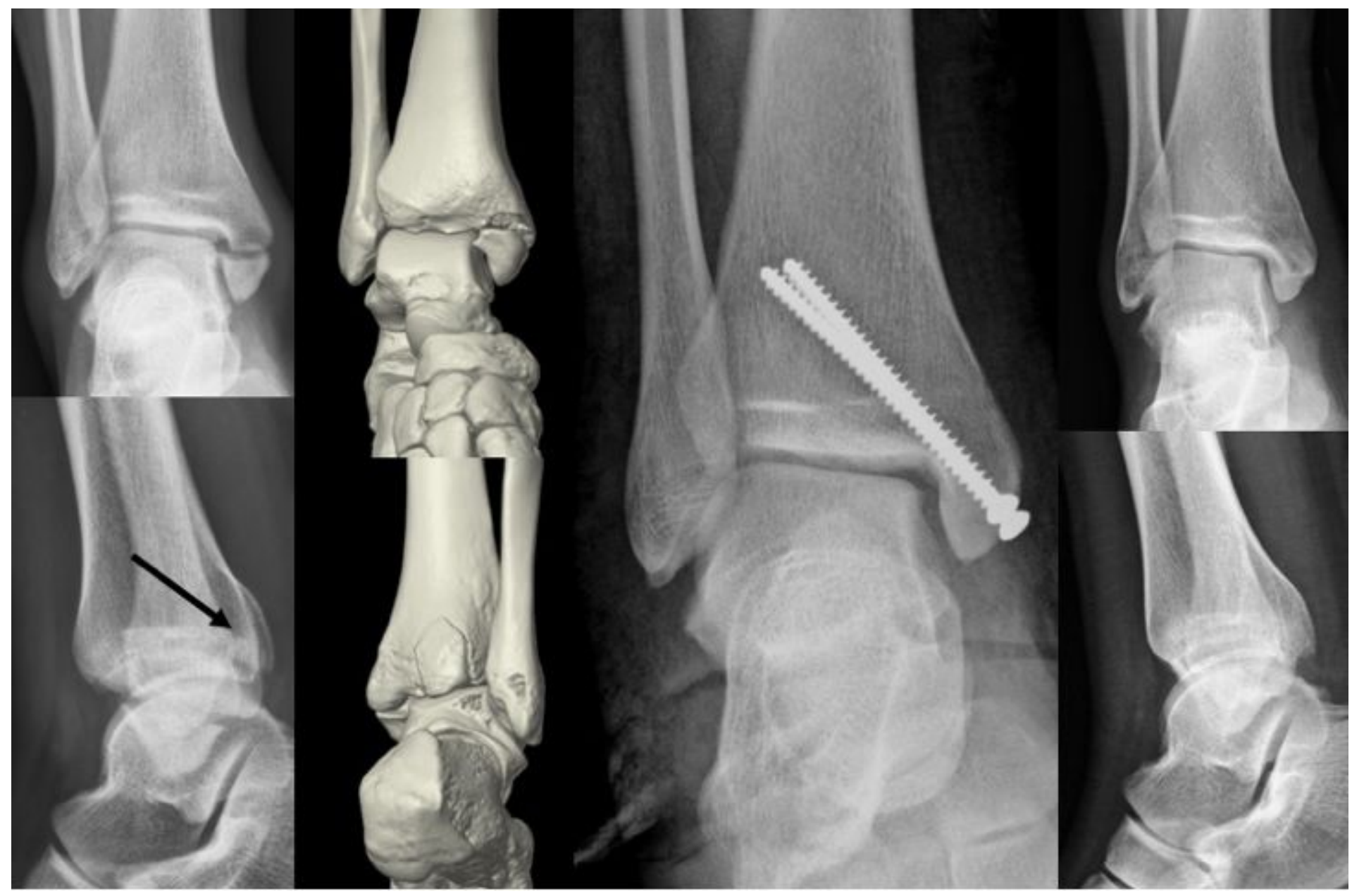

Figure 5

A 35-year-old man sustained a right isolated medial malleolar fracture due to motorcycle accident. A stage 2 pronation-abduction type was diagnosed based on the avulsion tear of posterior inferior tibiofibular ligament (black arrow). Three-dimensional computed tomography (3-D CT) confirmed the ligament avulsion. Cancellous screws were inserted for stabilizing fractured medial malleolus. A good ankle function was achieved for 1.5-year follow-up. 


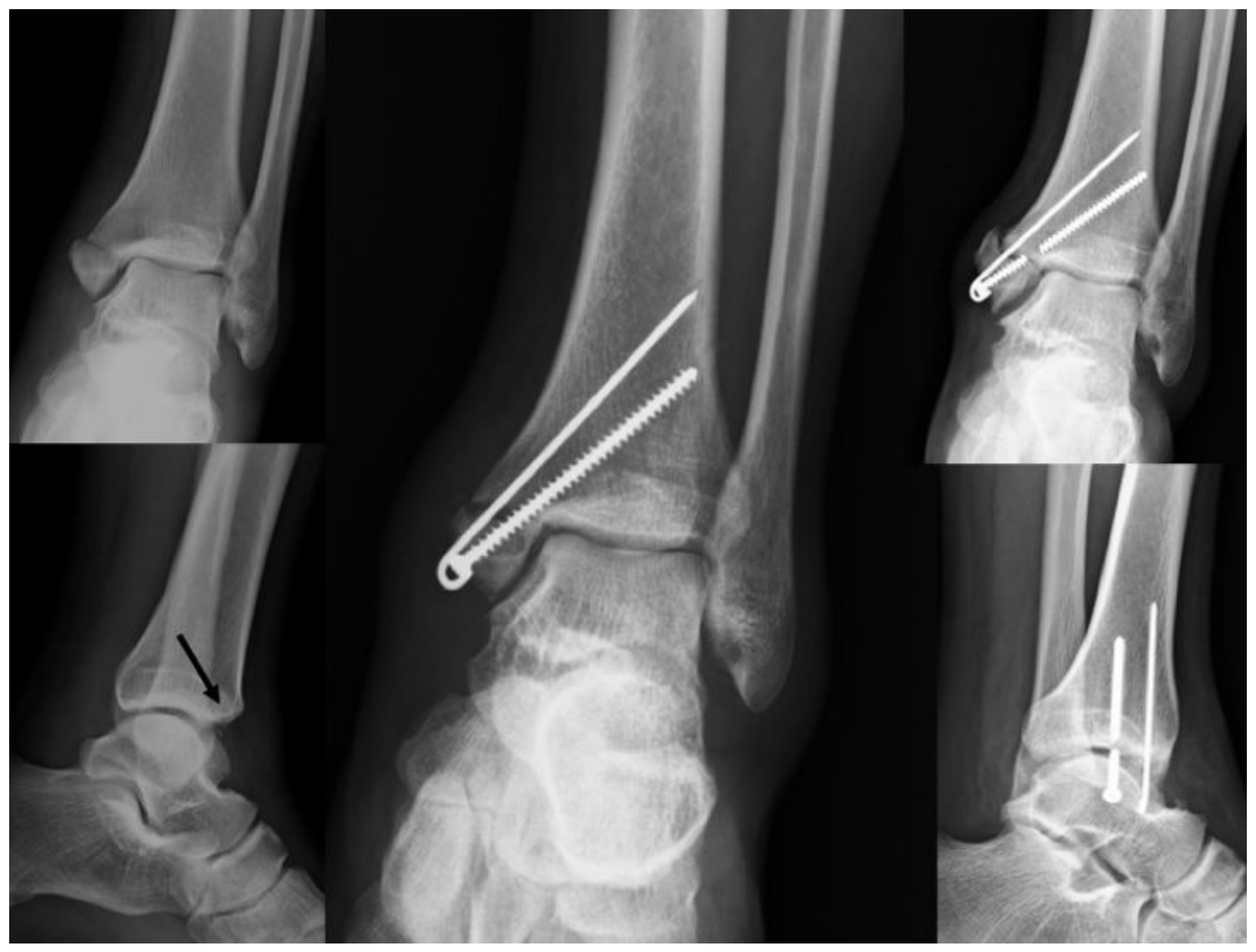

Figure 6

A 46-year-old man sustained a left isolated medial malleolar fracture due to heavy object compression. A stage 2 pronation-external rotation type was diagnosed based on avulsion tear of anterior inferior tibiofibular ligament (black arrow). Cancellous screws were inserted for stabilizing fractured medial malleolus. Screw breakage occurred at 7 months and nonunion persisted for 2-year follow-up.

\section{Supplementary Files}

This is a list of supplementary files associated with this preprint. Click to download.

- medmadata.xlsx

- medmadata.xlsx

- IRBankle.docx

- IRBankle.docx 